\title{
Patología placentaria en la etiología eclámpsica ${ }^{(*)}$
}

\author{
Doctor Víctor Merlano Merlano
}

Médico Residente. Bogotá, D. E. Clínica $1^{\circ}$ de Mayo.

En los últimos años la etiología de la toxemia se ha enfocado desde dos puntos de vista: primero por el hallazgo de necrosis placentaria y segundo por la influencia del espasmo vascular.

En 1930 R. A. Bartholomew descubrió en la cara materna de la placenta, áreas de degeneración reciente, especialmente en casos de "Abruptio". El hallazgo sugería más la causa que el efecto. ya que representaba tejido degenerado en contacto con la corriente sanguínea materna intervellosa; por lo tanto con posibilidad de diseminar en la circulación general de la madre productos tóxicos residuales.

Posteriormente, realizando exámenes sistemáticos macro y microscópicamente de placentas fijadas en formol, tanto de pacientes normales como de toxémicas, se relacionaron tales datos con los hallazgos clínicos. Muy pronto fue fácil afirmar que las áreas degenerativas placentarias eran encontradas en las pacientes toxemicas en un 85 a $90 \%$ de los casos, y raramente en placentas de pacientes normales. Por último se encontró una gran variedad de lesiones, siendo la más frecuente el infarto placentario.

De esta manera, y teniendo en cuenta las historias clínicas de las pacientes, fue posible conocer y clasificar el grado de necrosis placentaria. Se encontró desde uno de evolución lenta e inespecífico para la toxemia que se denominó A. B.; casos específicos para toxemia que se llamaron C. D. y E.; hasta un grado F. G. con pérdida de sustancia y necrosis placentaria. Todo:s estos hallazgos constantes, aomo expresión fisiopatológica del infarto.

(*) Tesis de grado, 28 de octubre de 1958. Universidad Javeriana. Declarada MERITORIA. Extractada por el doctor M. A. Fernández Bastidas. 
La especificidad de los tipos agudos de infartación placentaria por preeclampsia y eclampsia han sido estudiados por Patterson, Hunt Thousen y últimamente en nuestro país por Henao-Cabal y Muñoz-Delgado.

El fracaso de otros investigadores para verificar tales hallazgos se debió a que examinaron las placentas todavía frescas, no fijadas en formol (las lesiones en placentas frescas están ocultas o enrarecidas por la sangre); quedando pues establecido que para poder identificarles es indispensable fijar previamente el material en formalina.

Revisando la literatura de antes de 1930, encontramos que Young en 1914 ya hablaba de las lesiones encontradas en las placentas de las toxémicas; sin embargo afirmó que tales lesiones eran el resultado de anormalidades en la circulación materna.

El intento en obtener hoy día más del 85 al 90\% de correlación entre el diagnóstico clínico de la toxemia gravídica y los hallazgos placentarios, puede explicarse en parte por la posibilidad de que la toxemia llegue a desarrollarse en un periodo comprendido entre la fecha de última consulta prenatal y la iniciación del trabajo. Los casos que ilustran esta posibilidad se encontraron con frecuencia en nuestro estudio.

Por otra parte, un diagnóstico de toxemia basado en el análisis profundo no puede invalidarlo. El descubrimiento de que se desarrollaba en mayor grado, pero en forma ocasional, una proteinuria durante el trabajo de parto, constatada en un $10 \%$ de las pacientes ingresadas al hospital con orina y tensión arterial normal y presente en el $41 \%$ de ellas, ha sido descrita por Douglas, Morton y Chesley; en otras pacientes en ausencia de toxemia se ha encontrado aumento apreciable de la tensión arterial diastólica en la finalización del parto y en el primer día del puerperio. En otros casos, en fin, no se presenta ni proteinuria ni hipertensión durante el trabajo, invalidando las teorías que quieren explicar el fenómeno simplemente por el esfuerzo mismo que significa el parto.

Adoptando la teoría etiológica de la necrosis placentaria, debemos puntualizar que el examen microscópico de las placentas ha mostrado en casos comprobados de toxemia zonas negras u oscuras, características y bien definidas de infartación tipo E. Que en general tales áreas son más oscuras en este grupo que en los demás y por último da la impresión, que el mismo factor desencadenante del trabajo, puede también por un mecanismo parecido, des- 
arrollar infartación placentaria y motivar un acceso de toxemia intra-partum.

\section{PATOLOGIA PLACENTARIA ESPECIFICA DE LA TOXEMIA DEL EMBARAZO}

Está tan bien correlacionado al factor necrosis que ha llegado a afirmarse que valiéndose de cortes de placenta fijadas en formol al $10 \%$ durante tres o cuatro semanas se puede aclarar si una paciente dada es o no toxémica. Por tal sistema es igualmente posible determinar si la toxemia es gradual o aguda o si se presenta como "Abruptio". Las convulsiones ocasionales pueden presentarse poco antes de iniciarse el trabajo o un poco después de finalizado el parto, acompañadas de una ligera proteinuria o sin ella y con aumento de la presión sanguínea. Puede pensarse, durante estas convulsiones, más bien en epilepsia que en eclampsia. En tales casos dudosos el examen anatomopatológico de la placenta ofrece el único medio de diagnóstico para aceptar o descartar la eclampsia.

Hasta hoy los obstetras y patólogos no han reconocido el valor del conocimiento dado por el estudio anatomopatológico de la placenta, tanto como medio para afirmar o negar la presencia de la toxemia, como para diferenciar la hipertensión de la toxemia de la hipertensión por enfermedad vascular.

Debemos mencionar que muchos autores consideran la infartación placentaria como el efecto más bien que la causa de la gravi-toxemia. Este punto de vista reconoce que la necrosis placentaria es el resultado de una toxina desconocida que se halla presente en la circulación materna. Ahora, de acuerdo con un concepto más aceptado, se considera que el resultado de la isquemia placentaria se debe a arterioesclerosis o a una estenosis de las arteriolas deciduales. Teniendo en consideración la estructura de la placenta y el curso de la circulación intervellosa, estos puntos de vista han caído en desuso.

\section{CURSO DE LA CIRCULACION INTERVELLOSA MATERNA}

La placenta se compone de cotiledones parcialmente separados por tabiques deciduales; 'estos últimos no se extienden más de dos tercios de distancia de la decidua del corion calvo, dejando así un espasmo libre de red vellosa del corion calvo que permite la intercomunicación entre todos los cotiledones, por lo tanto los coti- 
ledones no pueden considerarse como unidades aisladas con su propia salida y entrada de sangre materna, sino como agrupación con conexiones mutuas.

La circulación sinusoidal de la sangre materna intervellosa, que la suministran las arteriolas deciduales, se halla impulsada por la presión sanguínea materna, haciéndola subir al corion, nivel en el cual cambia de dirección y sigue circulando periféricamente hasta el borde de la placenta. En este punto del corion placentario, la decidua refleja y el amnios se extienden por sobre el borde de la placenta para convertirse en corion calvo o decidua verdadera y amnios extraembrionario, respectivamente. Se adhieren a la pared uterina $u$ obstruyen el desplazamiento periférico de la sangre materna, formando así un seudo-canal llamado seno marginal, que circunscribe los bordes de la placenta. Se forman comunicaciones entre el seno marginal y las venas uterinas subyacentes que devuelven la sangre a la circulación general.

Rommey sostiene que el tabique decidual tiene arteriolas maternas funcionales en su porción placentaria que permiten la salida de la sangre a la parte superior de los espacios intervellosos, punto en el cual la sangre desciende para salir de la placenta por las venas deciduales en la unión deciduo-placentaria.

Los cortes efectuados en tejido placentario tomados a varios niveles descartan por completo el concepto anterior.

Los troncos vellosos se caracterizan por un tapizado epiteliocoriónico, estroma muscular-fibroso y por la presencia de vasos vellosos funcionales. Los tabiques deciduales se caracterizan por ausencia de epitelio coriónica y en cambio presentan células deciciuales en forma de una sustancia homogénea igual a la hialina.

En la placenta con necrosis se observan imágenes de vasos al microscopio con paredes hialinizadas y residuos amorfos, por tanto no parece que la sangre materna se descargue en la cima del tabique decidual para dirigirse a la decidua basal con el fin de encontrar salida del cotiledón por orificios venosos maternos de la decidua.

Como las arteriolas deciduales maternas también descargan sangre a la zona deciduo-placentaria, un doble riego sanguineo produciría movimientos paradójicos de la sangre arterial simultáneamente en direcciones opuestas. Además, tomando un útero con feto y placenta e inyectando un colorante en las arterias uterinas, se ve que dicho colorante se dirige desde las arterias deciduales por espacios intervellosos a la placa coriónica para luégo dirigirse 
a los senos marginales, volviendo a la circulación general por las comunicaciones existentes con las venas uterinas .

El colorante inyectado en las venas uterinas sigue una dirección inversa.

Si fuera posible que cualquier arteriola decidual se obstruyera o por cualquier motivo se obliterara, entonces los vellosos dependientes o secundarios podrían ser abastecidos por la corriente sanguínea subcoriónica aun cuando la sangre a este nivel fuese de calidad inferior. Por tanto, en vista de la trayectoria de la circulación placentaria, un factor necrosante presente en la sangre materna debe necesariamente afectar toda la placenta, produciendo necrosis generalizada. Por el contrario, el examen de placentas en casos de preeclampsia y eclampsia, han mostrado que la necrosis tiene un carácter local preponderante que se limita a la zona de una o más venas vellosas obstruídas, lo cual da lugar a una gran dilatación de los capilares vellosos, formando arborizaciones y los espacios intervellosos obliterados que cubren el área de distribución de las venas bloqueadas. Esto explica solamente el hecho de que los vasos vellosos que eran normales se encuentren bordeados por arborizaciones vellosas necrosadas de una área infartada adyacente sin la intervención del tabique decidual.

La necrosis total placentaria se observa en "Abruptio" fulminante y en la toxemia que se inicia durante el trabajo con las características del grupo E. En tales casos los espasmos difusos que afectan los vasos de la cara fetal de la placenta, hacen creer que los esfínteres de casi todas las venas placentarias de esta cara 0 de la sustancia placentaria se hallan en estado de espasmo.

\section{ESPASMO VASCULAR}

Explica el mecanismo de la isquemia infarto-placentario. Hoy es discutible todavía si el término infarto puede aplicarse a áreas de necrosis vellosa.

Una área de necrosis de tejido placentario resultante de la oclusión de una vena placentaria fetal, trae como consecuencia el abultamiento de los vasos vellosos secundarios; disminución de los espacios sanguíneos intervellosos y trombosis del riego sanguíneo materno intervelloso, denominándose todo este conjunto, infarto, con tanta propiedad como si se tratara de necrosis de una área pulmonar en la cual un proceso embólico la privara del riego sanguíneo arterial. 
Al iniciar este estudio postulamos la teoría de que la trombosis proviene posiblemente del impacto vigoroso de los movimientos fetales sobre los vasos superficiales de la placenta. Más tarde, al descubrir las células grasosas subendoteliales, se sugirió la posibilidad de un cambio ateromatoso y la formación de trombos en los vasos fetales, explicándose en forma poco satisfactoria el infarto placentario.

Pero las investigaciones de Spanner fueron decisivas para expilcar la infartación placentaria. Por cortes seriados y ciertos métodos de inyección, no sólo demostró el curso de la circulación materna intervellosa y la existencia de senos marginales, sino también la presencia de esfínteres en las venas fetales como en las venas del tejido placentario. La función fisiológica de estos esfínteres no se conoce bien, pero sí se puede aceptar que sobre ellos actúa una hormona espasmogénica que es la causante del espasmo produciéndose consecuencialmente impedimento parcial o total en la salida de sangre de la unidad placentaria.

Si observamos la cara fetal de la placenta de una paciente toxémica, inmediatamente después del primer trimestre, notaremos numerosos espasmos, lo cual contrasta con lo que se encuentra en la misma cara de la placenta en pacientes normales durante la gestación y el parto. El espasmo tardío de las arterias y venas fetales que se observa en la superficie placentaria después del parto, generalmente se consideran de poca importancia y se atribuyen a anoxia de los vasos inmediatamente después del desprendimiento de la placenta.

En un estudio hecho sobre 100 placentas al final del primer trimestre del embarazo para investigar el espasmo vascular, desechando algunas de ellas porque el examen no se hizo inmediatamente, se encontró que 56 placentas $(61 \%)$ no mostraron espasmo. En 6 de ellas ( $70 \%$ ) hubo pre-eclampsia leve al iniciarse el parto, sin embargo el espasmo del tejido placentario no fue bien demostrable. Sólo se encontró espasmo vascular marcado en 36 de estas placentas, las cuales correspondían a preclampsias graves. Quizás con más casos se hubieran obtenido mejores resultados.

El corazón fetal envía sangre continuamente a las zonas placentarias obstruídas, lo cual hace que los capilares se ingurgiten y distiendan con abultamiento y arborización vellosa consecuenciales, disminución y obliteración de los espacios intervellosos y suspensión de la circulación intervellosa materna, cuya vitalidad depende del epitelio coriónico. Se presenta luégo la necrosis del 
epitelio, que puede fácilmente reconocerse por picnosis, cariorexis y finalmente por cariolisis del núcleo. Este proceso representa una forma indirecta de infarto en que el funcionamiento normal de los canales arteriales intervellosos maternos dependen por completo del tamaño de los vasos vellosos, tamaño que asímismo depende del grado de espasmo y poder obstructivo de los esfínteres venosos.

Los vasos vellosos de la Mola Hidatiforme, lo mismo que los de la eritroblastosis edematosa, se arborizan y abultan, produciendo ocasionalmente un efecto que puede dar lugar a la toxemia.

A grandes rasgos, el área infartada de la placenta aparece un tanto más gruesa por el ensanchamiento de los innumerables vasos vellosos y mucho más oscura, por trombosis intervellosa e intracapilar, debida a necrosis del epitelio coriónico y del endotelio capilar velloso, por estasis de la circulación en tal área.

Lo que se denomina "Infarto Rojo" deriva su nombre del hecho de que los capirales vellosos se rompen y la sangre extravasada forma un coágulo dentro de la zona infartada por incompatibilidad con la sangre materna,

El "Infarto Negro" se llama así por su color oscuro, ya qu@ está formado por sangre trombosada de color oscuro y se presenta un poco antes del desprendimiento de la placenta o durante el período de expulsión de la misma. En el "Abruptio" agudo y en las pacientes que desencadenan la toxemia en el trabajo, se encuentran zonas extensas de infartos placentarios que interesan múltiples esfinteres. La pre-eclampsia grave y la eclampsia se caracterizan por infartos placentarios oscuros y localizados del Grupo E tantas veces mencionado; pero dichos infartos también pueden ser ligeramente claros, cuando interesan en menor grado a los esfínteres.

En la preeclampsia leve y sostenida se encuentra, en gran porcentaje de casos, el infarto $\mathrm{D}$, de color pardo debido a los cambios en la hemoglobina; el infarto $\mathrm{C}$, de color grisáceo, que se presenta en las preeclampsias leves de evolución lenta y es causado por la oclusión parcial de los esfínteres; el infarto D, color amarillo, y el infarto A, color amarillo blancuzco, no tienen etiología tóxica, siendo debido aparentemente a un proceso obliterativo lento de la arteria fetal correspondiente, lo cual paulatinamente ocasiona trombosis y hialinización de la sangre intervellosa, dando lugar a los "vasos vellosos extraños". La ausencia de ingurgitación y distensión de los capirales en los "vasos vellosos extraños" 
indica que el proceso ha sido de evolución lenta, atóxico y que se inicia sin espasmos de los esfínteres.

La doctora Zeek ha descrito áreas pálidas, redondas u ovaladas que se presentan ocasionalmente en las placentas de toxémicas. Dichas áreas han sido denominadas por ella con el nombre de "Isquemia Nodular", debido a que los capilares vellosos muestran un menor contenido de sangre. No hay distensión de los vasos vellosos; los espacios intervellosos son de tamaño normal, el epitelio coriónico y el endotelio capilar no presentan signos de necrosis. Se cree que la "Isquemia Nodular" es causada por espasmos de la arteriola placentaria fetal, siendo por lo tanto la antítesis de los infartos tóxicos de los grupos $\mathrm{E}$ y $\mathbf{D}$, ya que éstos se originan por espasmos del esfínter venoso. Es posible que el agente espasmogénico causante de cada uno de estos tipos de infarto sea el mismo.

Con el objeto de aclarar conceptos, hacemos un resumen de los principales hechos demostrados en placentas toxémicas:

1) Hay una patología placentaria específica en la forma de infarto placentario agudo y subagudo, que se demuestra mejor en placentas fijadas en formol, en las pacientes con preeclampsia, eclampsia y "abruptio placentae".

2) Las áreas necrosadas de placentas en contacto con la circulación intervellosa maternal presentan posibilidades de diseminación de los productos de autolisis placentaria, los cuales son altamente tóxicos para la madre.

3) Según Bartholomew, el infarto placentario de tipo agudo y subagudo es lógicamente la causa y no el efecto de la toxemia en la preñez. La interrelación de los componentes maternos y fetales de la circulación placentaria llevan a esta conclusión.

4) La base anatómica del infarto placentario se encuentra en la presencia de esfínteres en las venas placentarias.

5) El estímulo que excita al espasmo de los esfínteres venosos es lógicamente la oxcitocina, la cual, por los cambios en el balance hormonal al final de la preñez (baja de progesterona y aumento en la concentración de estrógenos libres), es aparentemente libertada de la inhibición ejercida por sus hormonas antagónicas, o sea la progesterona y la pitocinaza. El aumento de desoxicorticosteroides al final del embarazo, ayudados por el sodio, sensibiliza las paredes de los vasos y probablemente la de los esfínteres de la vena placentaria para aumentar su reactividad. 
6) El espasmo de los esfínteres venosos obstruye la salida de sangre fetal de los cotiledones y causa distensión de los capilares vellosos, abultamiento de los vasos vellosos, disminución u obliteración de los espacios intervellosos y además necrosis por anoxia del epitelio coriónico y del endotelio capilar.

7) La tromboplastina, abundante en el tejido placentario, es probablemente el primer producto de necrosis coriónica, la cual, si el infarto es extenso causa el "abruptio" por la formación de coágulos sub-placentarios y ocasionalmente afibrinogenemia con tendencia a la hemorragia.

8) Posiblemente existe una toxemia verdadera en la preñez, que es aquélla que se debe a infarto placentario y a ncrosis, como sucede con la preeclampsia, eclampsia y "abruptio placentae".

9) Se espera que el estudio bioquímico de placentas toxémicas y normales y de la sangre puedan servir para identificar los efectos y naturaleza de otros productos de degradación en la necrosis placentaria, los cuales seguramente tendrán más relación con la preeclampsia y la eclampsia.

10) Infarto A. Causado por la evolución lenta de una esclerosis gradual que abarca las arterias placentarias en la periferia de la placenta. No es de origen tóxico; sin embargo Bartholomew cree que da la voz de alerta cuando va a empezar la difusión de productos tóxicos en la circulación materna. Tiene color amarillo blancuzco, de consistencia firme por la hialinización de la sangre intervellosa y por la falta de circulación.

11) En una etapa más avanzada el infarto A da origen al infarto B, el cual se forma de una endarteritis obstructiva con hialinización de los canales sanguíneos intervellosos y presencia de células hemáticas degeneradas, dándole un color amarillo. No hay espasmos pero sí se observa neoformación de "vasos venosos extraños".

No es de causa tóxica, por lo tanto no se encuentra como factor etiológico de la toxema gravídica, pero, como en el caso anterior, también la anuncia.

12) El infarto $\mathbf{C}$ es motivado por el espasmo parcial de los esfínteres venosos e nlos casos de preeclampsia leve, de evolución sostenida por la difusión de guanidina e histamina en la circuulación materna. Se forma en tres o cuatro semanas, color grisáceo ćebido a que los aritrocitos están bañados en fibrina y se puede encontrar en cualquier parte de la placenta. 
13) El infarto D se presenta en la preeclampsia grave por acción espasmogénica marcada de oxcitocina sobre los esfínteres venosos, obstruyendo el $85 \%$ de la luz del vaso. Evoluciona en una c dos semanas, tiene color pardo por cambios en la hemoglobina, da necrosis placentaria acentuada y produce mayor cantidad de histamina y guanidina que en el caso anterior; sustancias que al llegar al torrente sanguíneo materno dan síntomas más severos de toxemia que puede desembocar en una eclampsia. Hay distención de las vellosidades y ruptura de los capilares terminales. Se presenta con más frecuencia en la cara uterina de la placenta.

14) El infarto E es de causa tóxica aguda y evoluciona rápidamente, en cuestión de horas, ocasionando en la placenta zonas extensas de necrosis de color negro y en forma difusa; este color negro se debe a la éstasis sanguínea venosa en las vellosidades, formando coágulos oscuros y a la sangre extravasada por la ruptura cie capilares. Se presenta e nel "abruptio placentae", que es de curso fulminante y en la toxemia que se desencadena durante el trabajo por espasmo repentino y total de los esfínteres venosos fetales sobre los cuales actúa drásticamente la oxcitocina.

La localización de los infartos es de tener en cuenta, pues de ella depende la vida del feto. Los más peligrosos son los que se presentan en la parte central de la placenta por las áreas de separación que ocasionan con extravasación de sangre; en cambio los infartos marginales apenas causan pequeñas separaciones que en nada alteran la oxigenación y nutrición fetales.

La ordenación literal de estos infartos no se debe propiamente a una diferencia etiológica fundamental, en lo cual están de acuerdo todos los investigadores, sino más bien a una gradación cie la gravedad en la evolución.

Cuanto más severa sea la toxemia, mayor será el número de infartos y cada uno de ellos tendrá un color más acentuado y una consistencia menos firme.

De acuerdo con los datos anteriores sobre la etiología de la preeclampsia, no hay duda de que los trastornos bioquímicos e histoquímicos de la placenta tienen primordial influencia, ya que hay pruebas que lo indican de esta manera.

Como bien lo dice Bartholomew, uno de los investigadores más ciestacados en la patología placentaria, a medida que se ha ido profundizando en el estudio del espasmo vascular y la necrosis placentaria, se han encontrado explicaciones a innumerables hechos hasta ahora desconocidos, que dan luz en el mecanismo del des- 
arrollo de la toxemia gravídica. Se ha comprobado que la placenta єs fuente de sustancias tóxicas, alérgicas, vasoconstrictoras y hormonales.

\section{MATERIAL}

Las placentas para este estudio fueron adquiridas en el Servicio de Toxemias de la Clínica Primero de Mayo entre las pacientes toxémicas puras que reunían todas las condiciones de diagnóstico y exámenes de laboratorio.

Después de un año de selección cuidadosa de las gestóxicas, reunimos 33 placentas, de las cuales once son de pre-eclampsia leve, doce de pre-eclampsia grave y diez de eclampsia. Se tuvo el cuidado de que el alumbramiento fuera lo más normal posible, con el fin de que los tejidos placentarios quedaran intactos y así poder hacer un examen macroscópico satisfactorio que dejara reconocer cualquier lesión patológica que nos sirviera para tomar de allí los cortes.

Las placentas que resultaron elegidas o coleccionadas en la forma expuesta anteriormente, las fijamos en formol al $10 \%$ durante 15 días; después, de cada placenta tomamos cuatro cortes de los sitios siguientes: 1) borde placentario; 2) cotilledón; 3) espacio inter-cotiloideo, y 4) porción proximal del cordón umbilical.

Estos cortes los tuvimos en formol al $10 \%$ por 48 horas y después por la técnica conocida los colocamos en hematoxilina-eosina.

Ya con las placas coloreadas procedimos a hacer un estudio metódico de cada una de ellas, es decir, examinamos las placas, las analizamos, practicamos diagnóstico y luégo hicimos correlación del dato anatomo-patológico con el clínico.

Encontramos que la lesión patológica más frecuente en las placentas es e linfarto; la arterioesclerosis, la hemorragia por ruptura de los capilares vellosos, la degeneración de las vellosidades y la infiltración leucocitaria polimorfonuclear en segundo término. Lesiones que están en relación proporcional con el tiempo de evolución clínica de la toxicosis gravídica. De ah íque encontramos en varios casos de pre-eclampsia leve una o dos de las lesiones anunciadas; en cambio en un caso de eclampsia de evolución rápida, de esas que se desarrollan en forma instantánea, no hay ninguna lesión característica de la fase aguda de la toxemia y sólo distinguimos en las placas congestión y edema. 


\section{PATOLOGIA PLACENTARIA}

PRE-ECLAMPSIA LEVE

\begin{tabular}{|c|c|c|c|c|c|c|c|c|}
\hline $\begin{array}{l}\mathrm{N} \text {. de } \\
\text { placa }\end{array}$ & Edad & $\begin{array}{c}\text { Diagnóstico } \\
\text { Clínico }\end{array}$ & $\begin{array}{c}\text { Edad } \\
\text { Embarazo }\end{array}$ & $\begin{array}{l}\text { Gra- } \\
\text { videz }\end{array}$ & T. A. & Edema & Proteinuria & Hallazgos patológicos \\
\hline $58-865$ & 30 & $\begin{array}{l}\text { Pre-eclamp- } \\
\text { sia leve }\end{array}$ & 40 & 7 & $17 \times 10$ & $\mathrm{x}$ & Huellas & Arterio esclerosis. Infarto D. \\
\hline $57-976$ & 36 & - & 38 & 7 & $15 \times 9$ & $\mathrm{xx}$ & Huellas & $\begin{array}{l}\text { Arterioesclerosis moderada. Infil- } \\
\text { tración leucocitaria polimorfonu- } \\
\text { clear. Calcificaciones. Infarto C. }\end{array}$ \\
\hline $57-1437$ & 27 & - & 40 & 5 & $16 \times 11$ & $\mathrm{xx}$ & $0.50 \mathrm{gm} \% \mathrm{o}$ & $\begin{array}{l}\text { Arterioesclerosis moderada. Infil- } \\
\text { tración leucocitaria polimorfonu- } \\
\text { clear. Degeneración de las vellosi- } \\
\text { dades. Infarto C. }\end{array}$ \\
\hline $57-1436$ & 28 & - & 22 & 6 & $16 \times 10$ & & $0.75 \mathrm{gm} \% \mathrm{o}$ & $\begin{array}{l}\text { Difusa infiltración polimorfonu } \\
\text { clear. Arterioesclerosis marcada. } \\
\text { Hemorragia. Infarto C. }\end{array}$ \\
\hline $58-508$ & 33 & - & 35 & 10 & $14 \times 9$ & & $0.36 \mathrm{gm} \% \mathrm{o}$ & $\begin{array}{l}\text { Esclerosis vasculas con zonas de } \\
\text { calcificación. Infarto } \mathbf{C} \text {. }\end{array}$ \\
\hline $58-519$ & 27 & - & 40 & 3 & $15 \times 8$ & & $0.21 \mathrm{gm} \% \mathrm{o}$ & $\begin{array}{l}\text { Arterioesclerosis moderada. Con- } \\
\text { gestión. }\end{array}$ \\
\hline
\end{tabular}


PRE-ECLAMPSIA LEVE

\begin{tabular}{|c|c|c|c|c|c|c|c|c|}
\hline $\begin{array}{l}\text { No de } \\
\text { placa }\end{array}$ & Edad & $\begin{array}{l}\text { Diagnóstico } \\
\text { Clínico }\end{array}$ & $\begin{array}{c}\text { Edad } \\
\text { Embarazo }\end{array}$ & $\begin{array}{l}\text { Gra- } \\
\text { videz }\end{array}$ & T. A. & Edema & Proteinuria & Hallazgos patológicos \\
\hline $28-521$ & 28 & $\begin{array}{l}\text { Pre-eclamp- } \\
\text { sia leve }\end{array}$ & 38 & 9 & $17 \times 10$ & & $1 \mathrm{gm} \% \mathrm{o}$ & $\begin{array}{l}\text { Zonas de necrosis por envejeci- } \\
\text { miento de las vellosidades y áreas } \\
\text { de calcificación. }\end{array}$ \\
\hline $58-864$ & 22 & - & 40 & 1 & $14 \times 9$ & & Huellas & $\begin{array}{l}\text { Arterioesclerosis marcada de los } \\
\text { capilares vellosos con calcificacio- } \\
\text { nes difusas. }\end{array}$ \\
\hline $58-789$ & 20 & - & 40 & 1 & $15 \times 9$ & & $0.40 \mathrm{gm} \% \mathrm{o}$ & $\begin{array}{l}\text { Congestión caracterizada por va- } \\
\text { so dilatación y edema de los capi- } \\
\text { lares vellosos. Zonas hemorrágicas. }\end{array}$ \\
\hline $58-824$ & 22 & - & 40 & 1 & $17 \times 10$ & & Huellas & $\begin{array}{l}\text { Necrosis moderada con infiltra- } \\
\text { ción leucocitaria polimorfonuclear. } \\
\text { Congestión vascular y hemorragia. }\end{array}$ \\
\hline $57-1516$ & 24 & - & 40 & 1 & $15 \times 10$ & $x x$ & $0.64 \mathrm{gm} \% \mathrm{o}$ & $\begin{array}{l}\text { Depósitos de calcio. Arterioescle- } \\
\text { rosis moderada. Congestión y he- } \\
\text { morragia }\end{array}$ \\
\hline
\end{tabular}


PRE-ECLAMPSIA GRAVE

\begin{tabular}{|c|c|c|c|c|c|c|c|c|}
\hline $\begin{array}{l}\text { Ne de } \\
\text { placa }\end{array}$ & Edad & $\begin{array}{l}\text { Diagnóstico } \\
\text { Clínico }\end{array}$ & $\begin{array}{l}\text { Edad } \\
\text { Embarazo }\end{array}$ & $\begin{array}{l}\text { Gra- } \\
\text { videz }\end{array}$ & T. A. & Edema & Proteinuria & Hallazgos patológicos \\
\hline $57-977$ & 42 & $\begin{array}{l}\text { Pre-eclamp- } \\
\text { sia grave }\end{array}$ & 40 & 7 & $17 \times 11$ & $\mathrm{xx}$ & $0.90 \mathrm{gm} \% \mathrm{o}$ & $\begin{array}{l}\text { Congestión vascular. Hemorra- } \\
\text { gia. Edema. Arterioesclerosis y cal- } \\
\text { cificaciones. Infarto C. }\end{array}$ \\
\hline $58-825$ & 27 & - & 37 & 7 & $16 \times 12$ & & $1 \mathrm{gm} \% \mathrm{o}$ & $\begin{array}{l}\text { Arterioesclerosis. Areas hemo- } \\
\text { rrágicas. Infiltración leucocitaria } \\
\text { polimorfonuclear. Infarto C. }\end{array}$ \\
\hline $58-814$ & 32 & - & 30 & 6 & $18 \times 12$ & $\mathrm{xxx}$ & $3.67 \mathrm{gm} \% \mathrm{o}$ & $\begin{array}{l}\text { Arterioesclerosis marcada. Con- } \\
\text { gestión y hemorragia. }\end{array}$ \\
\hline $58-866$ & 25 & - & 40 & 1 & $17 \times 10$ & $\mathrm{xxx}$ & $0.72 \mathrm{gm} \% \mathrm{o}$ & $\begin{array}{l}\text { Hemorragia por ruptura de los } \\
\text { capilares vellosos. Congestión y ede- } \\
\text { ma de las vellosidades. }\end{array}$ \\
\hline $58-863$ & 29 & - & 40 & 1 & $16 \times 10$ & $\mathrm{xxx}$ & $8.64 \mathrm{gm} \% \mathrm{o}$ & $\begin{array}{l}\text { Arterioesclerosis marcada. Necro- } \\
\text { sis y calcificaciones de las vellosi- } \\
\text { dades. Hemorragia intensa y con- } \\
\text { gestión. }\end{array}$ \\
\hline $58-862$ & 26 & - & 38 & 1 & $14 \times 10$ & $x x$ & $5,76 \mathrm{gm} \% \mathrm{o}$ & $\begin{array}{l}\text { Esclerosis de los capilares vello- } \\
\text { sos. Depósitos de calcio en los es- } \\
\text { pacios inter-vellosos. Congestión } \\
\text { vascular y hemorragia. }\end{array}$ \\
\hline
\end{tabular}


PRE-ECLLAMPSIA GRAVE

\begin{tabular}{|c|c|c|c|c|c|c|c|c|}
\hline $\begin{array}{l}\text { No de } \\
\text { placa }\end{array}$ & Edad & $\begin{array}{c}\text { Diagnóstico } \\
\text { Clínico }\end{array}$ & $\begin{array}{c}\text { Edad } \\
\text { Embarazo }\end{array}$ & $\begin{array}{c}\text { Gra- } \\
\text { videz }\end{array}$ & T. A. & Edema & Proteinuria & Hallazgos patológicos \\
\hline $58-861$ & 25 & $\begin{array}{l}\text { Pre-eclamp- } \\
\text { sia grave }\end{array}$ & 40 & 3 & $20 \times 10$ & $\mathrm{xxx}$ & $2.40 \mathrm{gm} \% \mathrm{o}$ & $\begin{array}{l}\text { Arterioesclerosis marcada. De- } \\
\text { pósitos de calcio. Congestión y he- } \\
\text { morragia. }\end{array}$ \\
\hline $58-788$ & 20 & - & 36 & 1 & $18 \times 10$ & $\mathrm{xxx}$ & $2.88 \mathrm{gm} \% \mathrm{o}$ & $\begin{array}{l}\text { Necrosis extensas. Infiltración } \\
\text { leucocitaria polimorfonuclear. He- } \\
\text { morragia y congestión. }\end{array}$ \\
\hline $57-1087$ & 30 & - & 36 & 4 & $16 \times 9$ & $\mathrm{xxx}$ & $3.06 \mathrm{gm} \% \mathrm{o}$ & $\begin{array}{l}\text { Calcificaciones abundantes. Ar- } \\
\text { terioesclerosis. Infiltración leucoci- } \\
\text { taria polimorfonuclear. Necrosis. }\end{array}$ \\
\hline $57-967$ & 26 & - & 30 & 1 & $17 \times 10$ & $\mathrm{xxxx}$ & $5.04 \mathrm{gm} \% \mathrm{o}$ & $\begin{array}{l}\text { Trombosis. Infiltración inflama- } \\
\text { toria de las paredes vasculares. In- } \\
\text { filtración leucocitaria polimorfo- } \\
\text { nuclear. Infarto D. }\end{array}$ \\
\hline $57-1624$ & 28 & - & 29 & 4 & $17 \times 12$ & $\mathrm{xxx}$ & $2.88 \mathrm{gm} \% \mathrm{o}$ & $\begin{array}{l}\text { Necrosis marcada. Calcificación. } \\
\text { Trombosis. Infarto D. }\end{array}$ \\
\hline $57-1517$ & 24 & - & 40 & 1 & $17 \times 10$ & $\operatorname{xxxx}$ & $2.40 \mathrm{gm} \% \mathrm{o}$ & $\begin{array}{l}\text { Arterioesclerosis. Calcificaciones. } \\
\text { Hemorragia y congestión de las ve- } \\
\text { llosidades. Infarto D. }\end{array}$ \\
\hline
\end{tabular}




\section{ECLAMPSIA}

\begin{tabular}{|c|c|c|c|c|c|c|c|c|}
\hline $\begin{array}{l}\text { No de } \\
\text { placa }\end{array}$ & Edad & $\begin{array}{c}\text { NDiagnóstico } \\
\text { Clínico }\end{array}$ & $\begin{array}{c}\text { Edad } \\
\text { Embarazo }\end{array}$ & $\begin{array}{l}\text { Gra- } \\
\text { videz }\end{array}$ & T. A. & Edema & Proteinuria & Hallazgos patológicos \\
\hline $57-1088$ & 27 & Eclampsia & 29 & 1 & $17 \times 11$ & $\operatorname{xxxx}$ & $5.76 \mathrm{gm} \% \mathrm{o}$ & $\begin{array}{l}\text { Necrosis extensas. Grandes de- } \\
\text { pósitos de calcio. Infiltración leu- } \\
\text { cocitaria polimorfonuclear. Infar- } \\
\text { to D. }\end{array}$ \\
\hline $57-1665$ & 22 & - & 28 & 2 & $17 \times 12$ & $\operatorname{xxxx}$ & $6.48 \mathrm{gm} \% \mathrm{o}$ & $\begin{array}{l}\text { Arterioesclerosis marcada. Trom- } \\
\text { bosis. Infarto D. }\end{array}$ \\
\hline $57-1145$ & 23 & - & 30 & 3 & $20 \times 12$ & $\operatorname{xxxx}$ & $7.2 \mathrm{gm} \% \mathrm{o}$ & $\begin{array}{l}\text { Arterioesclerosis marcada. Areas } \\
\text { de calcificación. Trombosis. Infar- } \\
\text { to D. }\end{array}$ \\
\hline $57-1144$ & 21 & - & 31 & 2 & $18 \times 10$ & $\operatorname{xxxx}$ & $10.8 \mathrm{gm} \% 0$ & $\begin{array}{l}\text { Arterioesclerosis marcada. Grandes } \\
\text { áreas de necrosis y clasificaciones } \\
\text { Infarto C. }\end{array}$ \\
\hline \multirow[b]{2}{*}{$58-513$} & \multirow[b]{2}{*}{19} & \multirow[b]{2}{*}{-} & \multirow[b]{2}{*}{40} & \multirow[b]{2}{*}{1} & \multirow[b]{2}{*}{$17 \times 11$} & \multirow[b]{2}{*}{$\mathrm{xxx}$} & \multirow[b]{2}{*}{$0.57 \mathrm{gm} \% \mathrm{o}$} & $\begin{array}{l}\text { Grandes depósitos de calcio. Ar- } \\
\text { terioesclerosis marcada. Infarto A. }\end{array}$ \\
\hline & & & & & & & & \multirow{2}{*}{$\begin{array}{l}\text { Extensas zonas de necrosis. Infil- } \\
\text { tración leucocitaria polimorfonu- } \\
\text { clear. Arterioesclerosis. Hemorra- } \\
\text { gia y congestión. }\end{array}$} \\
\hline $58-826$ & 29 & - & 28 & 3 & $18 \times 10$ & $\mathrm{xx}$ & $5.30 \mathrm{gm} \% \mathrm{o}$ & \\
\hline
\end{tabular}




\section{ECLAMPSIA}

\begin{tabular}{|c|c|c|c|c|c|c|c|c|}
\hline $\begin{array}{l}\text { No de } \\
\text { placa }\end{array}$ & Edad & $\begin{array}{l}\text { NDiagnóstico } \\
\text { Clínico }\end{array}$ & $\begin{array}{c}\text { Edad } \\
\text { Embarazo }\end{array}$ & $\begin{array}{l}\text { Gra- } \\
\text { videz }\end{array}$ & T. A. & Edema & Proteinuria & Hallazgos patológicos \\
\hline $57-1625$ & 31 & Eclampsia & 34 & 5 & $16 \times 11$ & $\mathrm{xxx}$ & $2.16 \mathrm{gm} \% \mathrm{o}$ & Hemorragia y congestión. \\
\hline $58-239$ & 18 & - & 40 & 1 & $14 \times 9$ & $\mathrm{xxxx}$ & $2.88 \mathrm{gm} \% \mathrm{o}$ & $\begin{array}{l}\text { Arterioesclerosis marcada. Gran- } \\
\text { des depósitos de calcio. Hemorragia } \\
\text { intensa. }\end{array}$ \\
\hline $57-1605$ & 20 & - & 37 & 1 & $18 \times 15$ & $\mathrm{xxxx}$ & $5.04 \mathrm{gm} \% \mathrm{o}$ & $\begin{array}{l}\text { Extensas zonas de necrosis. Arte- } \\
\text { rioesclerosis marcada de las vellosi- } \\
\text { dades. Calcificación. Infarto E. }\end{array}$ \\
\hline $57-968$ & 28 & - & 30 & 3 & $15 \times 10$ & $\mathrm{xxx}$ & $34.56 \mathrm{gm} \% \mathrm{o}$ & $\begin{array}{l}\text { Infiltración inflamatoria de las } \\
\text { paredes vasculares. Infiltración leu- } \\
\text { cocitaria polimorfonuclear. Arterio- } \\
\text { esclerosis. Extensas zonas de necro- } \\
\text { sis. Infarto E. }\end{array}$ \\
\hline
\end{tabular}




\section{RESUMEN DE LOS CUADROS ANTERIORES POR DATO CLINICO Y DE LABORATORIO}

1) Edad de las pacientes estudiadas:

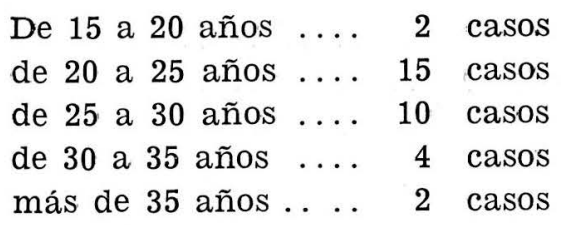

2) Diagnóstico clínico
a) Pre-eclampsia leve .. $\quad . . \quad 11$ casos o sea ..... $\quad . \quad 33.33 \%$
b) Pre-eclampsia grave ... 12 casos o sea ..... $36.33 \%$
c) Eclampsia $\ldots \begin{array}{lllllllllllll} & \ldots & \ldots & & 10 & \text { casos o sea } \ldots & \ldots & \ldots & 30 & \%\end{array}$

3) Edad del embarazo:

de 7 meses 11 casos o sea .... $33.33 \%$

de 8 meses 8 casos o sea .... $24.2 \%$ de 9 meses 14 casos o sea ... $42.4 \%$

4) Número de los embarazos o gravidez:
a) Primi $\ldots \ldots \ldots \quad \ldots 14$ casos
b) Secundi ..... 2 . 2 casos
c) Terci $\ldots \begin{array}{lllll} & \ldots & 5 & 5 & \text { casos }\end{array}$
d) Multi $\ldots \begin{array}{lllll} & \ldots & \ldots & 12 & \text { casos }\end{array}$

5) Indice de tensión arterial máxima:
De 14 a $16 \ldots \ldots \ldots \ldots$ casos
a) Pre-eclampsia leve
de $\quad \begin{array}{lllllllll} & 17 & \ldots & \ldots & \ldots & 3 & \text { casos }\end{array}$ de 16 a $18 \ldots \ldots \ldots . .96$ casos de $\quad 14 \ldots \ldots \ldots . .1$ caso

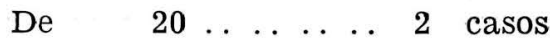
b) Pre-Eclampsia grave de $14 \ldots \ldots \ldots \ldots 1$ caso

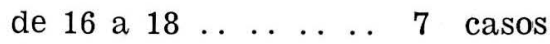
- Eclampsia
De 18 a $20 \ldots \ldots \ldots \ldots .1$ caso
de $\quad 15 \ldots \ldots \ldots .1$ caso 
6) Indice de la tensión arterial mínima:

\begin{tabular}{|c|c|c|c|c|c|c|c|c|c|}
\hline & De & 9 & . & .. & - . & .. & $\ldots$ & 4 & casos \\
\hline \multirow{3}{*}{ Pre-eclampsia leve } & de & 10 & . & . & $\ldots$ & $\cdots$ & . & 5 & casos \\
\hline & $\mathrm{de}$ & 11 & . & . & $\ldots$ & . & . & 1 & caso \\
\hline & de & 8 & . & . & $\cdots$ & . & . & 1 & caso \\
\hline \multirow{3}{*}{ Pre-eclampsia grave } & $\mathrm{De}$ & 10 & . & . & . & $\ldots$ & . & 7 & casos \\
\hline & de & 11 & . & . & $\ldots$ & . & . & 2 & casos \\
\hline & de & 12 & . & . & . & . & . & 3 & casos \\
\hline \multirow{5}{*}{ Eclampsia } & $\mathrm{De}$ & 9 & . & $\ldots$ & $\ldots$ & $\ldots$ & . & 1 & caso \\
\hline & de & 10 & $\ldots$ & $\ldots$ & $\ldots$ & $\ldots$ & . & 3 & casos \\
\hline & $\mathrm{de}$ & 11 & $\ldots$ & $\ldots$ & $\ldots$ & $\ldots$ & . & 3 & casos \\
\hline & $\mathrm{de}$ & 12 & $\ldots$ & . & $\ldots$ & . & . & 2 & casos \\
\hline & $\mathrm{de}$ & 15 & $\ldots$ & $\ldots$ & $\ldots$ & $\ldots$ & $\ldots$ & 1 & caso \\
\hline
\end{tabular}

7) Frecuencia de ediema maleolar:

a) Pre-eclampsia leve .. .... 3 casos.

b) Pre-eclampsia grave ..... Todos los casos

c) Eclampsia . . . . . . . . . . . . Todos los casos

8) Proteinuria:

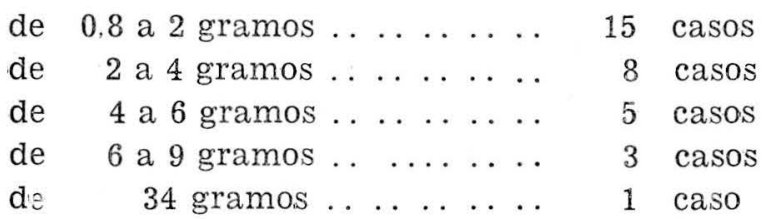

9) Hallazgos de anatomía patológica:

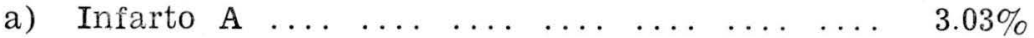
b) Infarto $\mathrm{C} \ldots \ldots \ldots \ldots \ldots \ldots \ldots \ldots$

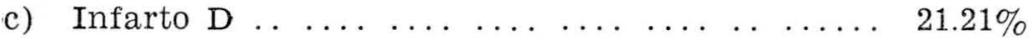

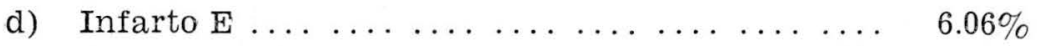

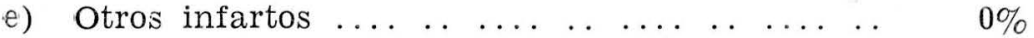

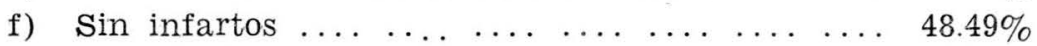

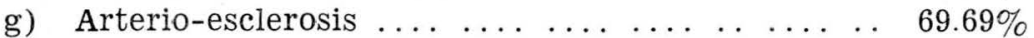

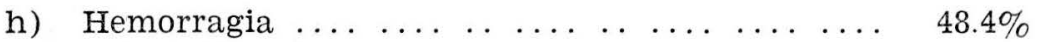

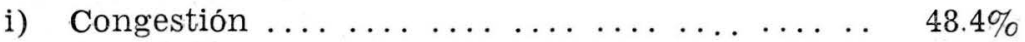

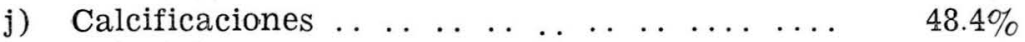
k) Infiltración leucocitaria polimorfonuclear .. . . $30.3 \%$
1) Degeneración de las vellosidades . . . . . . . . $27.2 \%$
m) Necrosis por envejecimiento de las vellosidades $3.03 \%$ 


\section{COMENTARIO Y CONCLUSIONES}

Haciendo un breve comentario de las lesiones microscópicas encontradas en las 33 placentas de pacientes toxémicas, examinadas y el orden descendente en que las colocamos según frecuencias, tenemos:

1) Arterio-esclerosis. - Se nota en la gran mayoría de las placentas. Su intensidad varía desde una moderada pérdida del tejico elástico de las arterias hasta la verdadera fibrosis en que las arterias se notan como tubos rígidos.

2) Infarto placentario.- -Se encuentra en casi todas las placentas. De todos los tamaños, llegando algunos a abarcar grandes zonas que se notan isquémicas, necrosadas con manifiesta picnosis, cariorrexis y cariolisis en los núcleos de las células de las vellosidades.

Como etiología de ellos encontramos la trombosis, el vasoespasmo arterial y venoso y en algunos casos la pared vascular ocluída por infiltración inflamatoria.

3) Hemorragia.-De hallazgo frecuente. Se vio claramente que era motivada por ruptura de los capilares vellosos por dilatación. Esta dilatación capilar produce ensanchamiento de la vellosidad, uniendo unas con las otras de tal manera que en muchos cortes no se encontraron espacios intervellosos. Estos sitios son susceptibles de hemorragia.

4) Zanas de calcificación.-Se ven difusas en las vellosidades. Én algunos casos de pre-eclampsia encontramos grandes depósitos de calcio. En ambos casos se notaron signos de isquemia.

5) Congestión vascular.-Se observa en las paredes de los vasos infiltración inflamatoria, moderada vaso-dilatación y edemas

6) Infiltración leucocitaria polimorfonuclear.-Fue hallada con mucha frecuencia, lo cual es de importancia, pues no ha sido descritas por los anatomo-patólogos ni se encuentra consignada en la literatura que hasta ahora se conoce sobre la histo-patología olacentaria. Hísticamente esta infiltración leucocitaria polimorfonuclear se puede atribuir a una reacción secundaria motivada por la muerte de los tejidos. 
7) Degeneración de las vellosidades.—Se notan pálidas y desintegradas por oclusión parcial de los capilares vellosos e isquemia moderada de los tejidos.

8) Necrosis por envejecimiento de las vellosidades.-Estas necrosis no se atribuyen a infartos sino a simple desintegración ceiular por caducidad.

9) Trombosis.-De grandes y pequeños vasos, causando los infartos.

10) Edemas de las vellosidades.-Quizá por dificultad en la circulación venosa de retorno.

\section{FORMAS DE INFARTOS EN LOS CASOS CLINICOS}

a) Pre-eclampsia leve.-En estas placentas se pudo diagnosticar dos tipos de infartos de los cuales habla Bartolomew en sus investigaciones y cuyos conceptos se tuvieron en cuenta para este estudio. Ellos son:

Infarto C: cuatro casos: (57-1436; 57-1437; 57-976 y 58-508).

Infarto D: un caso (58-865).

Es decir, cinco casos de infarto en 11 placentas.

b) Pre-eclampsia grave.-Se investigaron 12 placentas, de las cuales 5 resultaron con infartos tipo $\mathrm{C}$ y $\mathrm{D}$, distribuídos en la siguiente forma:

Infarto C: 2 casos: 58-825 y 57-967.

Infarto D: 3 casos, a saber: 57-1517; 57-1624 y 57-967.

No hubo infarto $\mathrm{E}$, tan encontrado por algunos autores.

.Eclampsia.-7 infartos encontrados en un total de 10 placentas Infarto A: un caso, o sea 58-513.

Infarto C: un caso, que es el 57-1144.

Infarto D: tres casos, o sean 57-1088; 57-1665 y 57-1145.

Infarto E: dos casos, que son 57-968 y 57-1605.

Del estudio anterior se deduce que el infarto es una lesión patológica característica de las placentas de pacientes toxémicas, siendo los más frecuentes los de tipos C y D. Es de anotar también que las arterias trombosadas tenían marcados procesos de esclerosis, presumiendo que esta lesión vascular es la fase inicial de los infartos, y en proporción insignificante contribuye a la oclusión vascular la infiltración inflamatoria y la vaso-constricción. 
Esta última es quizá la causa más eficiente de la toxicosis gravídica.

Este trabajo investigativo sobre el complejo problema de la eclamptogenia debe considerarse como una prueba más de que las lesiones placentarias más frecuentes en las toxémicas, tales como el infarto, la necrosis, la isquemia y la degeneración, son hallazgos típicos como ya lo habían descrito Bartholomew, Thomsen, etc., y que además es de tenerse en cuenta la arterio-esclerosis como lesión frecuentísima, según queda demostrado en nuestro trabajo. Nos hace dudar es si estas lesiones aparecen primero que el cuadro clínico toxémico, porque como ya dijimos antes, en un caso de eclampsia de transcurso rápido (es decir, sin pródromos de ninguna clase), sólo se observa hemorragia y congestión. Este hecho es muy sugestivo, hasta el punto que hacemos hincapié en él porque en realidad las mayores lesiones placentarias fueron las encontradas en las pacientes cuyas toxicosis eran de evolución lenta. Seguramente la causa de la eclampsia en esta paciente fue el espasmo vascular generalizado en la placenta y motivdo por una sustancia desconocida, que ha podido ser la oxcitocina, que luégo pasó a la circulación materna, desencadenando la gestosis. Pero como el parto se verificó en pocas horas, esa vaso-constricción o "esa sustancia" no alcanzó a producir lesión hística.

Este hecho nos hace pensar que no siempre la prueba etiología de la toxicosis gravídica está en la patología placentaria, sino que hay otra causa desconocida relacionada con el tiempo y severidad con que evolucionó la intoxicación materna.

Nuestra consideración se apoya también en otro caso de eclampsia de evolución un poco más lenta que el ya comentado, en que se encontró arterio-esclerosis y hemorragia, pero no hubo ni infarto ni necrosis.

Creo que cuando se perfeccione la técnica ideada por Bartholomew y se tenga especial cuidado en la fijación de los especímenes y la adquisición de los cortes en las zonas en donde el aspecto macroscópico lo haga más aconsejable, será factible hacer el diagnóstico del tipo clínico de la toxemia con placentas de procedencia desconocida, porque la severidad de la toxicosis deja huella inconfundible en el tejido placentario; reconocible fácilmente al microscopio por el número de infartos y su tipo que están así en relación directa con el tiempo de evolución y gravedad de la paciente.

Como conclusiones del presente estudio, tenemos: 
a) En cuanto a la patología placentaria, como etiología de Ja toxicosis gravídica, encontramos algunas lesiones de las ya descritas por Bartholomew en sus investigaciones, lo cual acredita este trabajo, y otras que aun cuando no han sido descritas tienen singular importancia porque contribuyen a sustentar con más énfasis lo dicho de que la toxemia se acompaña de trastornos patológicos del tejido placentario. No consideramos que esos trastornos son la causa primitiva de la toxemia, sino la consecuencia de una causa desconocida que generalmente transcurre lentamente, pero que otras veces es de aparición rápida y aguda, como sucedió en los casos de que trata nuestro trabajo; porque no de otra forma se puede explicar que unas placentas de pacientes eclámpsicas tengan lesiones anatomo-patológicas y otras no.

Además, ha quedado comprobado que la histo-patologia está de acuerdo con la evolución de la enfermedad, lo cual sugiere que las lesiones a medida que avanzan van constituyendo la sintomatología toxémica porque seguramente en esos tejidos necrosados c isquémicos se producen sustancias tóxicas que favorecen los trastornos histológicos y a la vez contribuyen al cuadro clinico materno.

b) La infiltración leucocitaria polimorfonuclear, hallazgo no descrito en otras investigaciones, en un 30.3\% corrobora la existencia de necrosis.

c) La necrosis por envejecimiento de las vellosidades, hallazgo no descrito en otras investigaciones, denota degradación de las células, seguramente por acción de sustancias tóxicas.

d) Encontramos arterio-esclerosis en el $69.69 \%$ de los casos, porcentaje más alto que el infarto, y por lo tanto hallazgo de gran importancia, quizá debida a la hipercolesterolemia que generalmente tienen estas pacientes. 


\section{BIBLIOGRAFIA}

1. BARTHOLOMEW, R. A. and COLVIN, B. D.-Diagnosis of the ocurrence of toxemia of pregnancy by examination of the unknown placenta.

2. WOODBURY, R. A., HAMILTON, W. F. and TORPIN, R.-The Relationship between Abdominal, Uterine and Arterial Pressures during Labor, Am. J. Physiol, 1938, 121.640.

3. EASTMAN, N. J.-Foetal Blood Studies, I The Oxygen Relationships of Umbilical Cord Blood at Birth. Bull, J. Hop. 1930, 47, 221.

4. PLASS, E. D. and MATTHEWS, C. W.-Placental Transmission, III Amino Acids, Non Protein Nitrogen, Urea and Uric Acid in Fetal and Mater nal Whole Blood Plasma and Corpuscles.

5. BELL, W. B., CONNUNGHAM, L., JOWETT, M., Millet, H. and BROOKS, J.-The Metabolism and Acidity of the foetal Tissues and Fluids.

6. SLEMMONS, J. M. and STANDER H. J.-The Lipoids of the Maternal anf Fetal Blood at the Conclusion of Labor.

7. NEEDHAM, J.-Chemical Embryol. Cambridge.

8. BOYD, E. M. and WILSON, K. M.-The Exchange of Lipids in the Umbilical Circulation at Birth.

9. DANN, W. J.-The Transmission of Vitamin A from Parents to Young in Mammals.

10. BRAESTRUP, P. W.-Studies of Latent Scurvy in Infants.

11. WALDSTEIN, E.--Zur Genese des Ovarialhormons.

12. CORNER, G. W. -The Sites of Formation of Eestrogenic Substances in the Animal Body Physiol. Rev., 1938, 18, 154.

13. SOXTON, J. and LEEB, L.-Thyroid-Stimulating and Gonadotropic Hormones of the Human Anterior-Pittuitary Gland. at Different Ages and in Pregnant and Lactating Women. Páginas 51,261.

14. WISLOCKI, G. B. and SNYDER, F. F.-Note on the Failure of Anterior Lobe Extract to Pass fron Fetus to Mother.

15. BECK, Alfred.-Práctica de Obstetricia. Páginas 53 y siguientes, 1953.

16. DEVRAIGNE, L.-Manual de Obstetricia. Páginas 35 y siguientes, 1943.

17. HENAO CABAL, MUÑOZ DELGADO.-Patología Placentaria. Rev. Col. de Obst. y Gine. 9:123, 1958.

18. FERNANDEZ BASTIDAS, M. A.-Patología Placentaria. Abruptio Placentae. Rev. Col. de Obst. y Ginec., 9:245, 1958. 